\title{
Epilepsy seizure types, classification and treatment
}

\author{
DS Magazi, S Nkohla, MT Mmako
}

Department of Neurology, Dr George Mukhari Academic Hospital, Sefako Makgatho Health Sciences University, Pretoria, South Africa *Corresponding author, email:dmagazi@profmagazi.co.za

\begin{abstract}
Epilepsy is a chronic condition whose building blocks are recurrent seizures. It is this varied presentation that at times poses a challenge to making a diagnosis. The response to treatment is also not uniform, making it necessary to individualise. This article discusses the various seizure types, the latest classification by the International League against Epilepsy (ILAE), treatment and prognosis of the condition.
\end{abstract}

Keywords: International League against Epilepsy, classification, seizure types, treatment

\section{Introduction}

Epilepsy is well described in ancient pagan and religious texts going by various names. One of its assigned names was the "sacred disease" since it was thought only a god could throw a man to the ground, rob him of his senses and revive him again. Hippocrates challenged the notion of the condition being of supernatural origins and instead suggested a scientific basis in a treatise called the Hippocratic Corpus. Whether he is the singular author of those documents is a debate that has never been resolved. ${ }^{1}$ Knowledge about epilepsy continues to grow with an ever-evolving understanding of how various aspects of it should be perceived or described.

The World Health Organization estimates that approximately 50 million people live with epilepsy worldwide, $80 \%$ being from low-income countries. ${ }^{2}$ The number of people who have presented with epilepsy to the department of neurology, Dr George Mukhari Academic hospital, a 1500 bed hospital serving the north-western townships of Pretoria, over a period of 10 years, is in excess of 2500 . It is thus the commonest condition seen in this department, forming a third of all neurological diagnoses.

\section{Seizures and recurrence}

The recurrence of various seizure types makes up the condition in that two or more of these, at least 24 hours apart, are a requisite for the definition in the strict sense of the word. This definition has been recently expanded to include a single seizure and a more than $60 \%$ chance of recurrence. ${ }^{3}$ The Multicenter Epilepsy and Single Seizure (MESS) study found the chances of a recurrence after a single unprovoked seizure in the group with a normal EEG and normal neurological findings to be in the order of $20 \%, 25 \%$ and $30 \%$ at 1, 2 and 4 years, respectively. ${ }^{4}$ A proportional hazards model testing chances of a recurrence showed hazard ratios for those with neurological abnormalities and abnormal electroencephalography (EEG) findings to be 1.35 (95\% Cl 1.07-1.72, $\mathrm{p}=0.013)$ and 1.54 (95\% Cl 1.27-1.86, $\mathrm{p}<0.0001)$, respectively. ${ }^{5}$ A prospective community-based cohort study showed first onset status epilepticus (SE) in children gives rise to further episodes of SE. ${ }^{6}$ To treat the select group of patients with first onset seizures reduces the chances of a recurrence.

The seizures that make up epilepsy do not have an immediately reversible cause (e.g. seizures from alcohol withdrawal, infections, electrolyte imbalance, etc). The practical importance of this realisation is the need to investigate someone with a first onset seizure for potentially dangerous causes as opposed to just treating for the chronic condition of epilepsy. Having corrected the underlying cause, the chances of recurrence over a 10 -year period is less in $80 \%$ of cases than if the seizures were unprovoked. ${ }^{7}$ There is an exponential increase for recurrence with second seizures and more giving credence to what the British neurologist, William Gowers said in 1881, "each attack facilitates the occurrence of another by increasing the instability of the nerve elements".8

\section{Seizure types}

Seizures are the clinical manifestations of an electrical "surge" which is cerebral in origin and can be broadly characterised as motor and non-motor. What governs the manifestation of the seizures is which part of the brain is the origin, e.g. occipital lobe seizures will present with visual phenomena and motor strip seizures would present with muscle jerks. The manifestations are further dictated by whether both cerebral hemispheres or just a part of the brain is the source of these seizures. 


\section{Motor seizures}

These are the most readily identifiable of the seizures, further divided into tonic, myoclonic, clonic, tonic-clonic, atonic, automatisms, hyperkinetic and epileptic spasms.

Tonic seizures: These seizures manifest with a spasm of the body with stiffened limbs and may even result in backward arching of the back followed by loss of consciousness. They tend to be brief, lasting seconds to at most a few minutes. Their isolated occurrence is a rare phenomenon seen especially in childhoodonset syndromes like the Lennox Gestaut. The aforementioned syndrome progresses into adolescence and adulthood. 9 Tonic seizures are a recognised manifestation of frontal lobe epilepsy especially originating in the supplementary motor cortex. ${ }^{10}$ A mimicker for these is a focal dystonia which would not affect consciousness and would not cause an abnormal ictal EEG.

Myoclonic seizures: These manifest as brief jerky movements of the muscle with preserved consciousness. Just like the tonic seizures, myoclonic seizures in isolation are not common, oftentimes coexisting with other seizure types and thus manifesting with loss of consciousness in those situations. There are, however, distinct epilepsy syndromes that present with myoclonus consistently like the juvenile myoclonic epilepsy (JME), progressive myoclonic epilepsies like Baltic myclonus/ Unverricht-Lundborg and mitochondrial cytopathies like MERRF (myoclonus epilepsy and ragged-red fibres). ${ }^{11,12,13}$ It is worth noting that myoclonus is not exclusively caused by epilepsy. The other forms include physiological myclonus, e.g. hiccups or hypnic jerks (brief jerks before falling asleep), essential myoclonus manifests with isolated myoclonus with minimal or no functional impairment and secondary myoclonus, e.g. the post anoxic action myoclonus of Lance Adams.

Clonic seizures: These are sustained muscle contractions which could have a focal or generalised presentation, depending on how much of the brain surface is involved.

Tonic-clonic seizures: This is the commonest form of the seizures with a tonic (stiffening) phase followed by a clonic (jerk) phase. Beyond the muscle stiffening and jerks is loss of consciousness, sphincter control and post ictal confusion. Unpublished data from our department of 2500 patients shows generalised tonic clonic seizures to be in the majority at $69 \%$ of presentations. This is echoed by Hamdy and co-workers from Saudi Arabia who found $76.2 \%$ of 341 patients with epilepsy to be having generalised tonic clonic seizures. ${ }^{14}$

Atonic seizures: These seizures are characterised by a loss of tone of the body with the patient falling to the ground in a limp form. It so happens that the loss of tone only affects a part of the body, notably the head with a head drop or nod. These are not common but are a distinct feature of the Lennox Gestaut syndrome. ${ }^{9}$

Automatisms: These are more or less coordinated movements associated with an ictus which occur with a disturbance of consciousness like walking aimlessly or being disruptive. There is usually an amnesia for these events when the patient comes around.
Hyperkinetic seizures: These are characterised by an agitated movement of a body part like pedalling of the feet.

Epileptic spasms: These are characterised by a sudden flexion of limb and truncal muscles and usually occur in series. They are a characteristic presentation of the West syndrome which manifests in infancy continuing to early childhood.

\section{Non-motor seizures}

These include absences, cognitive, emotional, sensory, autonomic and behavioural arrest.

Absence seizures: These are brief episodes (4-20 seconds mostly) of a halting of activities with a blank stare during which time the patient is not responsive. There may be some subtle motor phenomena like flickering of the eyes during an episode. Absences typically occur in childhood or adolescent age groups and have a generally good prognosis. ${ }^{15}$ This is true of typical absences which do not have post-ictal phenomena and are brief. The atypical presentations would include a longer duration absence (towards a minute and longer), post-ictal manifestations and when in combination with other seizure types like in JME and the Lennox Gestaut syndrome. These latter conditions, in contrast, generally have a poorer prognosis.

Cognitive and emotional seizures: A wide range of mental and psychological phenomena could be the sole or prominent presentation of certain seizures. These include illusions like out of body experiences, delusions and hallucinations (auditory, olfactory, tactile or visual). Further manifestations could include mood changes (fear, depression, anxiety, elation, etc) and forced thinking. The latter phenomenon are intrusive thoughts, for instance, a rapid recollection of past life experiences. There is a proposal that the term forced thinking be changed to "hypercognitive seizures".16 These seizures often have their origin in the temporal lobe, including the limbic structures even though, at times, the site of origin may be more diffuse. ${ }^{17}$

Sensory seizures: These seizures do not enjoy as much prominence as the motor manifestations in discussions of epilepsy. Their presentation, however, is not infrequent with the manifestations often being auras before motor phenomena. These include various hallucinations overlapping with what could have been categorised as cognitive. There could also be negative sensory phenomena with decreased appreciation of the specific senses. Peter Wolf aptly points out that the relation between epilepsy and sensation is bidirectional in that seizures could manifest with sensory phenomena and sensory stimuli in certain individuals could trigger seizures resulting in "reflex epilepsies".18 Examples of reflex epilepsies include seizures triggered by reading, taking hot showers, listening to the voice of particular radio presenters, etc. ${ }^{19}$

Autonomic seizures: These seizures manifest with sensations e.g. gastric phenomena like borborygmi, palpitations, piloerection causing "goose bumps", etc. Certain syndromes have autonomic manifestations as one of the defining features like the Panayiotopoulos syndrome which is an early onset epilepsy. There is a hypothesis about the possible underlying reason for 
SUDEP (sudden unexpected death in epilepsy) being partly from dysautonomia from compromised cardiorespiratory function. ${ }^{20}$

\section{Classification}

The diversity of seizures making up epilepsy make it necessary to look at epilepsy not as one monolithic group of conditions, but in fact diverse. To make sense of the diversity, a categorisation of these has become necessary. Ancient scholars including Galen in the second century recognised the need to classify epilepsy according to its various presentations. ${ }^{21}$ In recent times, the ILAE has been at the forefront of classifying epilepsy.22 The objective of classifying epilepsies is to make a precise characterisation of the semiology. This has the further spinoff of being able to decide on best treatment options. A further step beyond classifying the epilepsies is to identify recognisable syndromes and thus be able to further project on the long-term prognosis of a particular presentation.

The latest ILAE classification is based on whether the seizure is from a focal part of the brain or both cerebral hemispheres with the terms "focal onset" and "generalised onset" used for these, respectively. The focal onset is with undisturbed or impaired awareness. The latest classification factors in the instances where the clinician is unable to characterise the seizure and uses the term "unknown onset" and an "unclassified" group. In all three broad groups (focal, generalised and unknown onsets) there is a broad categorisation of the seizures into motor and non-motor with their various presentations, as discussed above. There is a further recognition of the group of patients who would have both focal and generalised onset seizures. Furthermore, a focal seizure may spread from a focus and become generalised, referred to as "focal to bilateral tonic clonic".22 Figure 1 below shows the basic layout of the ILAE classification. Hughlings Jackson described the phenomenon of a seizure "march" from a focal point to becoming generalised, interpreting this as a clinical analogue of spreading cerebral ictal activity. ${ }^{23}$ Factoring in co-morbidities and aetiologies (genetics, etc) with the aid of special investigations, facilitates the recognition of syndromes. These syndromes are a constellation of signs and symptoms which form a recognisable clinical entity.

\section{Challenges of diagnosing epilepsy}

Seizure detection and underreporting are not uncommon since there is a need to recognise the manifestations for what they are. ${ }^{24}$ The patient often has no insight about the phenomenology of the ictal events and the diagnosis is oftentimes based on an observer's narration of events which is sometimes a challenge, especially if the seizures are atypical. To counter this predicament is for visual recordings by the observer, a standard competence of most cellular phones to capture phenomena, to be shown to the attending practitioner.

Electroencephalography, a diagnostic tool for epilepsy, is sparsely distributed in many low-income countries with a concentration in the cities with resultant long waiting times. Affordability for the patient is a major challenge for many. ${ }^{25}$ Another compounding factor regarding EEGs is that not infrequently, lack of proper expertise has a potential to contribute to a high rate of erroneous results. ${ }^{26}$ The same applies to the availability of brain imaging facilities, including the CT scan and even more so, MRI (magnetic resonance imaging).$^{25}$

Competing culture-based/religious philosophies are widely prevalent in sub-Saharan Africa and also contribute to delayed diagnosis. Varying levels of stigma could also lead the patient and community not to embrace the diagnosis. ${ }^{27}$

\section{Management}

The aim of management is to reduce the frequency or eliminate the seizures completely. The following steps could be followed: 1) determine whether a phenomenon is a seizure or not; 2) determine whether the seizure has an immediate identifiable and correctable cause (provoked) OR epilepsy as discussed above; 3) classify and treat epilepsy, look for co-morbidities and workup for a cause for the condition; and 4) identify syndromes. It should be noted that $\mathrm{SE}$, provoked or with a background of the chronic condition of epilepsy, is an emergency. It is worth noting that a good description of the clinical presentation helps further in the assessment of EEGs and might be a reason to initiate therapy on its own merits. ${ }^{28}$

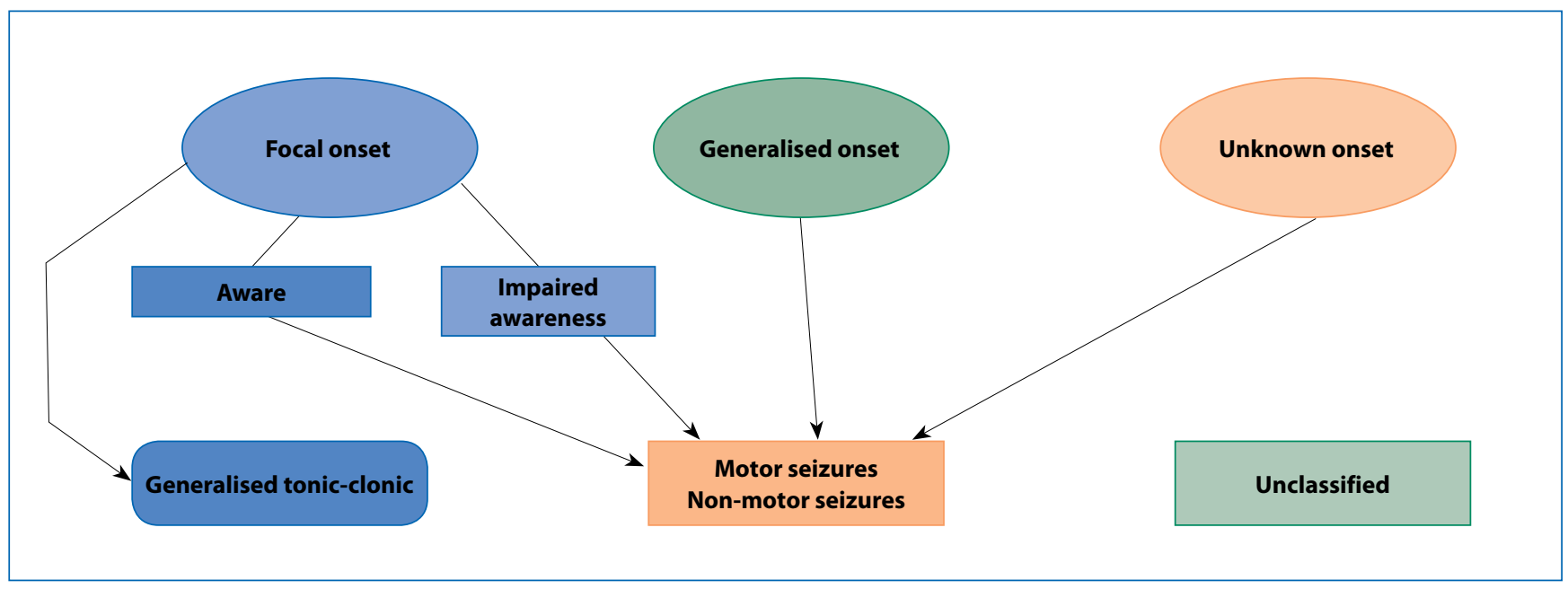

Figure 1. ILAE 2017 Classification of Seizure Types Basic Version (please refer to the various seizures types discussed above for detail) 
The antiepileptic drugs (AEDs) have varied mechanisms of action and thus are not uniformly efficacious for the different epilepsies; e.g. absence seizures don't respond to carbamazepine as opposed to ethosuximide, valproate or lamotrigine. ${ }^{29}$ Similarly, myoclonic seizures do not respond well to drugs like carbamazepine and phenytoin and respond well to valproate and levetiracetam. ${ }^{30}$ Valproate is a broad-spectrum AED and is recommended for generalised and unclassified epilepsies even though other AEDs can be efficacious in some patients. The authors, however, go on to caution about pregnancy and the risk of neural tube defects. ${ }^{31}$ The concern about neural tube defects from exposure of the unborn child can be minimised by using monotherapy at the lowest effective dose and the use of folic acid even though the effect of the latter measure is not as yet clear. ${ }^{32}$ Carbamazepine is regarded as the drug of choice for focal onset seizures but lamotrigine seems to be more efficacious for these. ${ }^{33}$ The "newer" AEDs including levetiracetam, gabapentin, lamotrigine, topiramate have been shown to be more tolerable with a lower frequency of clinical adverse effects as a result of less hepatic enzyme induction. ${ }^{34}$ Even though some newer AEDs are available in South African central hospitals, they are more readily available in the private sector. The efficacy of the older AEDs is undisputed, including phenobarb which is the cheapest AED on the market and is the sole drug in use in certain subSaharan countries.

Monotherapy is preferred to polytherapy as it brings down the cumulative side-effects that could otherwise manifest due to the simultaneous use of many drugs. Approximately two thirds of patients with epilepsy get reasonably controlled on monotherapy. ${ }^{35}$ Most anticonvulsants have to be titrated to the lowest effective and tolerable doses. This means that treatment should be tailor-made for the patient according to response. Epanutin is the only one with no such incremental titrations done. If monotherapy at adequate dosages does not yield the desired effect, a second drug can be introduced with later withdrawal of the first. ${ }^{36}$ For patients with resistant seizures, the practitioner could only then put the patient on polytherapy. A recording of the seizure frequency on a seizure diary by family or acquaintances could assist in determining control or lack thereof.

Approximately $20 \%$ of patients do not respond adequately on antiepileptic medication in spite of different treatment regimens. Having excluded the possibility of a wrong diagnosis, compliance problems, inadequate dosage, the epilepsy is then labelled intractable or refractory. The ILAE defines this as failed control in spite of two appropriately chosen AEDs. ${ }^{37}$ These patients could be assessed for suitability of other modalities including surgery and vagal nerve stimulation. ${ }^{38} \mathrm{~A}$ randomised controlled trial of patients with refractory epilepsy found effective seizure control in $58 \%$ of patients who had a temporal lobectomy compared to $8 \%$ on optimum medication. ${ }^{39}$ There is also an option to give the "ketogenic" diet to reduce seizures. ${ }^{40}$

Abrupt stoppage of AEDs is an important reason why people with epilepsy get SE. These are continuous seizures of more than five minutes or a cluster of shorter duration seizures without waking

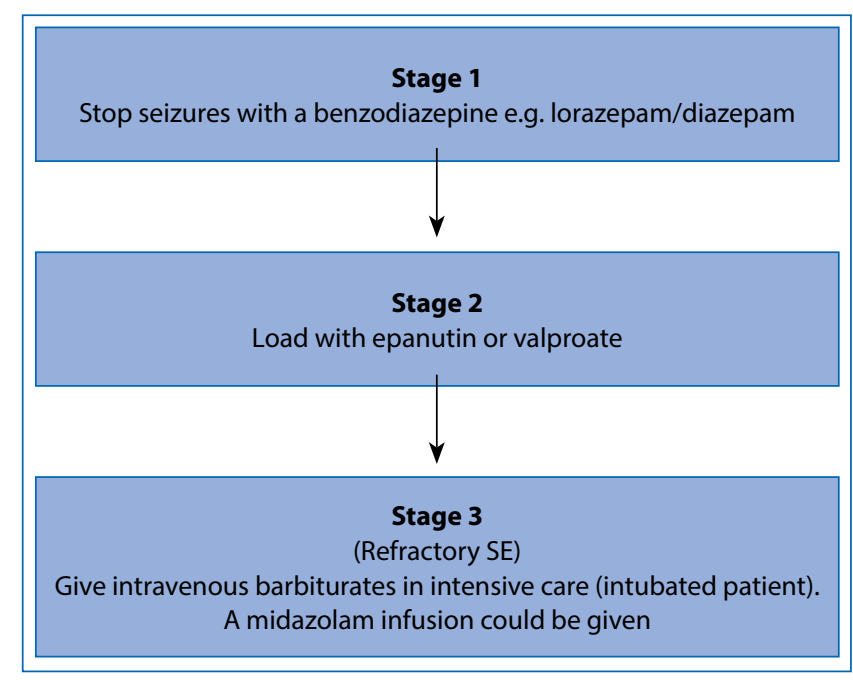

Figure 2. Stages of treatment of SE. Metabolic abnormalities, e.g. hypoglycaemia and acidosis should be considered and managed appropriately in addition

up in between. ${ }^{41}$ Figure 2 depicts a summary of the stages to be taken in managing $\mathrm{SE}$.

Stage 1: Stopping the seizure with a benzodiazepine like lorazepam (4-8 mg) IV/IM or diazepam (10-30 mg) IV. The rate should be half an ampoule per minute $(5 \mathrm{mg}$ per minute for diazepam and $2 \mathrm{mg}$ per minute for lorazepam) to minimise the chances of respiratory depression. Lorazepam has a much longer anticonvulsant effect than diazepam (12-24 hrs vs. 15-30 minutes respectively). Other administration routes have been employed e.g. buccal midazolam or rectal diazepam. ${ }^{42}$

Stage 2: The next step is to load with epanutin or valproate intravenously at $20 \mathrm{mg} / \mathrm{kg}$. Epanutin should be given in nonglucose containing solutions to avoid crystal formation at the rate of $50 \mathrm{mg}$ per minute. A faster infusion of intravenous epanutin carries a danger of the patient getting an arrest of cardiac function. ${ }^{43}$ Intravenous valproate could, on the other hand, be given faster since it is more "cardio-stable". The same loading dose could be repeated the second time if necessary for valproate and with epanutin, incremental doses of $5 \mathrm{mg} / \mathrm{kg}$ to a maximum of $30 \mathrm{mg} / \mathrm{kg}$ could be given.

Stage 3: Beyond the above measures, should the patient continue to be in SE, the management should be in intensive care for intubation and commencement of barbiturates which ordinarily would affect respiration. A barbiturate like phenobarb 15- $20 \mathrm{mg} / \mathrm{kg}$ could be given intravenously at a rate of $100 \mathrm{mg} / \mathrm{min}$. There are various other options of intravenous barbiturate preparations/regimens that could be employed at this stage for as long as the patient is intubated with respiratory support in an intensive care unit. A midazolam infusion is another mode of therapy in the intensive care setting that could be employed. Should the patient continue to have seizures or have a recurrence in spite of the above-mentioned measures beyond 24 hours, the diagnosis is "super refractory SE". Urgency of management cannot be overemphasised, ideally to control the SE within an hour or two. Refractory SE should be diagnosed with commencement of treatment at most within three hours. ${ }^{44}$ The more the delay in management of $\mathrm{SE}$, the greater the 
likelihood of not being able to achieve control with a resultant poorer prognosis. It should be noted that the management of SE has been dubbed "terra incognita" especially at the latter stages of management, meaning there is statistically robust evidence on the best way to manage hence a variability of firstly, the time frames of definition and management steps.

It should be noted that the management of epilepsy also entails education of the patient or caregiver about possible triggers of seizures like alcohol binges, the importance of compliance and the danger of not being compliant and what activities could be deemed dangerous. There should also be education of the caregiver/family to avert negative sentiments towards those living with epilepsy and to inform patients about local support groups. An organisation like "Epilepsy South Africa", an affiliate of the International Bureau for Epilepsy (IBE) plays an important role in supporting people living with epilepsy. South Africa is in need of expanded programmes of this nature that could reach even the smaller areas far out in the countryside.

\section{Treatment gap}

The treatment gap is defined as the percentage of people with active epilepsy requiring treatment but who are not on it. This is estimated at a staggering $75 \%$ in low-income countries, $50 \%$ in middle-income and only $10 \%$ in the high-income countries. ${ }^{45}$ A number of reasons for this include financial and other unique logistical challenges to getting treatment in poorer countries.

\section{Prognosis}

The outlook of epilepsy varies according to the cause or underlying mechanism. The overall prognosis, however, is that $60-70 \%$ of patients with newly-diagnosed epilepsy will become seizure free. ${ }^{46}$ Primary generalised epilepsy and a normal neurological examination, control of seizures with low-dose monotherapy are associated with a good prognosis. A meta-analysis of 1621 patients with refractory epilepsy who had undergone surgery showed a $44 \%$ achievement of seizure freedom. ${ }^{47}$ A proper selection of which patients can have surgery is a determinant of success.

Stopping medication should be at the recommendation of the attending practitioner and is usually in people who have been seizure-free for at least two years. It is done gradually rather than abruptly to reduce the chance of precipitating a seizure. The possibility of recurrence from stopping medication should be communicated with the patient and family, in which case, the medication should be resumed.

\section{Conclusions}

Seizures in epilepsy form a colourful tapestry of manifestations and because of their diverse nature might at times be difficult to recognise. Due to its chronicity and potentially distressing nature, the person living with epilepsy, family or caregivers could benefit from counselling. The patient living with epilepsy should know that epilepsy is not necessarily a lifelong sentence. The clinician therefore should be able to identify those with a good prognosis and empathetically shine light on the way forward for those with a poorer outlook.

\section{References}

1. van der Eijk PJ. The 'theology" of the Hippocratic treatise on the sacred disease. Apeiron. 1990;23(2): 87-119.

2. Geneva: World Health Organization; 2006. WHO. Neurological Disorders: Public Health Challenges.

3. Fischer RS, Bonner AM. The Revised Definition and Classification of Epilepsy for Neurodiagnostic Technologists. Neurodiagn J. 2018;58(1):1-10.

4. Kim LG, Johnson TL, Marson AG, Chadwick DW; MRC MESS Study group. Prediction of risk of seizure recurrence after a single seizure and early epilepsy: further results from the MESS trial. Lancet Neurol. 2006;5(4):317-22.

5. Marson A, Jacoby A, Johnson A, Kim L, Gamble C, Chadwick D, Medical Research Council MESS Study Group. Immediate versus deferred antiepileptic drug treatment for early epilepsy and single seizures: a randomised controlled trial. Lancet. 2005 Jun 11-17;365(9476):2007-13.

6. Berg AT, Shinnar S, Testa FM, et al. Status epilepticus after the initial diagnosis of epilepsy in children. Neurology. 2004;63(6):1027-34.

7. Hesdorffer DC, Benn EK, Cascino GD, Hauser WA. Is a first onset symptomatic seizure epilepsy? Mortality and risk for recurrent seizure. Epilepsia 2009;50(5):1102-8.

8. Gowers WR. (1881) Epilepsy and other chronic convulsive disorders: their causes symptoms and treatment. J \& A Churchill, London.

9. Vignoli A, Oggioni $G$, De Maria $G$, et al. Lennox-Gastaut syndrome in adulthood: Long-term clinical follow-up of 38 patients and analysis of their recorded seizures. Epilepsy Behav. 2017;77:73-78.

10. Jobst BC, Siegel AM, Thadani VM, et al. Intractable seizures of frontal lobe origin: Clinical characteristics, localising signs, and results of surgery. Epilepsia. 2000; 41(9):1139-52.

11. Baykan B, Wolf P. Juvenile myoclonic epilepsy as a spectrum disorder: A focused review. Seizure. 2017:49:36-41.

12. Genton $P$, Striano $P$, Minassian BA. The history of progressive myoclonus epilepsies. Epileptic Disord. 2016;18(S2):3-10.

13. Finsterer J, Zarrouk-Mahjoub S. Management of epilepsy in MERRF syndrome. Seizure. 2017;50:166-170.

14. Hamdy NA, Alamgir MJ, Mohammed el GE, et al. Profile of epilepsy in a regional hospital in Al qassim, Saudi Arabia. Int J Health Sci (Qassim). 2014;8(3):247-55.

15. Martínez-Ferrández C, Martínez-Salcedo E, Casas-Fernández $C$, et al. Long-term prognosis of childhood absence epilepsy. Neurologia. 2017 Mar 18. pii: S0213-4853(17)30021-X.

16. Stephani C, Koubeissi M. Hypercognitive seizures - Proposal of a new term for the phenomenon forced thinking in epilepsy. Epilepsy Res. 2017;134:63-71.

17. Elliott B, Joyce E, Shorvon S. Delusions, illusions and hallucinations in epilepsy: 1. Elementary phenomena. Epilepsy Res. 2009;85(2-3):162-71.

18. Wolf P. Epilepsy and the Sensory Systems. Epilepsy Curr. 2016; 16(6):369-372.

19. Wolf $P$, Koepp M. Reflex epilepsies. Epilepsy, Part I: Basic Principles and Diagnosis. (Stefan H, Theodore WH, ) Edinburgh: Elsevier, 2012: 257-276. Handbook of Clinical Neurology (Aminoff MJ, Boller F, Swaab DF, series eds.) vol 107.

20. Moseley B, Bateman L, Millichap JJ, et al. Autonomic epileptic seizures, autonomic effects of seizures, and SUDEP. Epilepsy Behav. 2013;26(3):375-85.

21. Temkin O. The Falling Sickness: A History of Epilepsy from the Greeks to the Beginnings of Modern Neurology. 1994; Volume 4 of Publications : 1st ser., Monographs, Johns Hopkins University Institute of the History of Medicine Softshell Books.

22. Brodie MJ, Zuberi SM, Scheffer IE, Fisher RS. The 2017 ILAE classification of seizure types and the epilepsies: what do people with epilepsy and their caregivers need to know? Epileptic Disord. 2018;20(2):77-87.

23. York GK 3rd, Steinberg DA. Hughlings Jackson's neurological ideas. Brain. 2011;134(Pt 10):3106-13.

24. Elger $\mathrm{CE}$, Hoppe C. Diagnostic challenges in epilepsy: seizure under-reporting and seizure detection. Lancet Neurol. 2018;17(3):279-288.

25. McLane HC, Berkowitz AL, Patenaude BN, et al. Availability, accessibility, and affordability of neurodiagnostic tests in 37 countries. Neurology. 2015; 85(18):1614-1622.

26. Birbeck GL. Epilepsy Care in Developing Countries: Part I of II. Epilepsy Curr 2010;10(4): 75-79. 
27. Kaddumukasa M, Kaddumukasa MN, Buwembo W, et al. Epilepsy misconceptions and stigma reduction interventions in sub-Saharan Africa, a systematic review. Epilepsy Behav. 2018;85:21-27.

28. Koutroumanidis M, Arzimanoglou A, Caraballo $R$, et al. The role of EEG in the diagnosis and classification of the epilepsy syndromes: a tool for clinical practice by the ILAE Neurophysiology Task Force (Part 1). Epileptic Disord. 2017;19(3):233-298.

29. Glauser T, Ben-Menachem E, Bourgeois B, et al. ILAE treatment guidelines: evidence-based analysis of antiepileptic drug efficacy and effectiveness as initial monotherapy for epileptic seizures and syndromes. Epilepsia. 2006;47(7):1094-120.

30. Caviness JN. Treatment of Myoclonus. Urotherapeutics. 2014; 11(1):188-200.

31. Marson AG, Al-Kharusi AM, Alwaidh M, et al; SANAD Study group. The SANAD study of effectiveness of valproate, lamotrigine, or topiramate for generalised and unclassifiable epilepsy: an unblinded randomised controlled trial. Lancet. 2007;369(9566):1016-26.

32. Yerby MS. Clinical care of pregnant women with epilepsy: neural tube defects and folic acid supplementation. Epilepsia. 2003; 44 Suppl 3:33-40.

33. Marson AG, Al-Kharusi AM, Alwaidh $M$, et al; SANAD Study group. The SANAD study of effectiveness of carbamazepine, gabapentin, lamotrigine, oxcarbazepine, or topiramate for treatment of partial epilepsy: an unblinded randomised controlled trial. Lancet. 2007;369(9566):1000-15.

34. French JA. New generation antiepileptic drugs: what do they offer in terms of improved tolerability and safety? Ther Adv Drug Saf. 2011;2(4): 141-158.

35. Kwan P, Brodie M. Early identification of refractory epilepsy. N Eng. J Med 2000;342(5):314-19.

36. St Louis EK, Gidal BE, Henry TR, et al. Conversions between monotherapies in epilepsy: expert consensus. Epilepsy Behav. 2007;11:222-234.
37. Sinha S, Siddiqui KA. Definition of intractable epilepsy. Neurosciences (Riyadh). 2011;16(1):3-9.

38. Mitchell JW, Seri S, Cavanna AE. Pharmacotherapeutic and Non-Pharmacological Options for Refractory and Difficult-to-Treat Seizures. J Cent Nerv Syst Dis. 2012;4: 105-115.

39. Wiebe S, Blume WT, Girvin JP, Eliasziw M. Effectiveness, efficiency of surgery for temporal lobe epilepsy study G. A randomized, controlled trial of surgery for temporal-lobe epilepsy. New Engl J Med. 2001;345 (5):311-8.

40. Dozières-Puyravel $B$, François $L$, de Lucia $S$, et al. Ketogenic diet therapies in France: State of the use in 2018. Epilepsy Behav. 2018. pii: S1525-5050(18)30345-7.

41. Lowenstein DH, Bleck T, Macdonald RL. It's time to revise the definition of status epilepticus. Epilepsia. 1999;40(1):120-122.

42. Nakken KO, Lossius MI. Buccal midazolam or rectal diazepam for treatment of residential adult patients with serial seizures or status epilepticus. Acta Neurol Scand. 2011;124(2):99-103.

43. Trinka E, Höller J, Leitinger M, Brigo F. Pharmacotherapy for status epilepticus. Drugs. 2015;75:199-1521.

44. Shorvon S, Ferlisi M. The treatment of super-refractory status epilepticus: a critical review of available therapies and a clinical treatment protocol. Brain. 2011;134(Pt 10):2802-18.

45. Meyer A-C, Dua T, Ma J, et al. Global disparities in the epilepsy treatment gap: a systematic review. Bull World Health Organ 2010;88:260-266.

46. Kwan P, Sander JW. The natural history of epilepsy: an epidemiological view. J Neurol Neurosurg Psychiatry 2004;75:1376-81.

47. Schmidt D, Staven K. Longterm seizure outcome of surgery versus no surgery for drug-resistant partial epilepsy: a review of controlled studies. Epilpesia 2009;50:1301-09. 